Asian Journal of Computer Science and Technology ISSN: 2249-0701 Vol.7 No.3, 2018, pp. 91-95

(C) The Research Publication, www.trp.org.in

\title{
Personalization of E-Learning Systems: Determination of Most Preferred Learning Style Using Conjoint Analysis
}

\author{
J. Saul Nicholas ${ }^{1}$ and F. Sagayaraj Francis ${ }^{2}$ \\ ${ }^{1}$ Research Scholar, Department of Computer Science and Applications, \\ SCSVMV University, Enathur, Kanchipuram, Tamil Nadu, India \\ ${ }^{2}$ Professor, Department of Computer Science and Engineering, Pondicherry Engineering College, Puducherry, India \\ E-Mail: saulnicholas@gmail.com,fsfrancis@pec.edu
}

\begin{abstract}
Identifying user preferences is a very important activity before offering a suggestion or a product. E-learning systems also follow suit in identifying the user preferences of learning style before offering the e-learning contents. There are several methods discussed in the literature for identifying the user preferences for e-learning contents. This paper presents a new method for the same purpose. The core of the new method is Conjoint Analysis, which is based on the type of the contents, preferred volume for each type of content and the ranking for the various combinations of the contents and their preferred volumes. The outcome of this method is the most preferred learning style of an individual learner.

Keywords: Adaptive Systems, Personalized Learning, Learning Styles, Adaptive Framework, Conjoint Analysis, FSLSM, Time Factor, Frequency Factor
\end{abstract}

\section{PREMISE OF CONJOINT ANALYSIS}

Conjoint analysis or preference analysis is a statistical method that started in mathematical psychology. Today it is widely used in many of the social sciences and applied sciences, including marketing, product management, operations research and many such domains [1].

Consider that a customer goes shopping to shop to buy a laptop. The shopkeeper offers different models with varying features like, RAM capacity, processor speed and price. Based on the choice, the customer can buy a laptop. In this the preference of the customer is very important. The preference for one of the alternatives will reveal the partworth utilities of individual attributes. In this example, the attributes are RAM capacity, processor speed and price [2, $3]$. When he chooses model A, it will show he put higher emphasis on RAM size. Choosing model B will reveal he gave higher emphasis for better processor. So in conjoint analysis, the part-worth utilities of individual attributes, in this case, RAM size, processor speed and price are calculated based on the selection or ranking for the defined set of combinations of attribute values $[4,5,6]$.

\section{CONJOINT ANALYSIS FOR PERSONALIZATION PARAMETERS}

From Felder and Silverman Learning Style Model (FSLSM) leading and widely used two attributes with three factors are considered as presented in Table I. They are, dimension 1 Active or Reflective, dimension 2 - Intuitive or Sensory, and dimension 3 - Visual or Verbal.
TABLE I CHOSEN ATTRIBUTES

\begin{tabular}{|c|c|c|}
\hline Factor & Attribute 1 & Attribute 2 \\
\hline Dimension 1 & Active & Reflective \\
\hline Dimension 2 & Sensory & Intuitive \\
\hline Dimension 3 & Visual & Verbal \\
\hline
\end{tabular}

Combination of all attributes with the different values will provide eight different options as given in Table II.

TABLE II MODEL AND THE VALUES

\begin{tabular}{|c|l|}
\hline Model & \multicolumn{1}{|c|}{ Attributes } \\
\hline Model 1 & Active, Sensory, Visual \\
\hline Model 2 & Reflective, Sensory, Visual \\
\hline Model 3 & Active, Sensory, Verbal \\
\hline Model 4 & Reflective, Sensory, Verbal \\
\hline Model 5 & Active, Intuitive, Visual \\
\hline Model 6 & Reflective, Intuitive, Visual \\
\hline Model 7 & Active, Intuitive, Verbal \\
\hline Model 8 & Reflective, Intuitive, Verbal \\
\hline
\end{tabular}

Converting this into a mathematical model, the levels are -1 and +1 for each level. Active is set as -1 and Reflective is set as +1 . Here the list of combinations with their coding is called the design matrix and is displayed in Table III. For $\mathrm{k}$ attributes, there are $2^{k}$ possible combinations [7]. A full factorial design is created making use of all possible combinations. It is given in Table III. The three attributes variables are treated as variables with the value of -1 to +1 .

TABLE III DESIGN MATRIX

\begin{tabular}{|c|c|c|c|}
\hline Model & $\begin{array}{c}\text { Dimension 1 } \\
(\mathbf{X 1})\end{array}$ & $\begin{array}{c}\text { Dimension 2 } \\
(\mathbf{X 2})\end{array}$ & $\begin{array}{c}\text { Dimension3 } \\
(\mathbf{X 3})\end{array}$ \\
\hline 1 & 1 & 1 & 1 \\
\hline 2 & 1 & -1 & -1 \\
\hline 3 & -1 & 1 & -1 \\
\hline 4 & 1 & 1 & -1 \\
\hline 5 & -1 & -1 & 1 \\
\hline 6 & 1 & -1 & 1 \\
\hline 7 & -1 & 1 & 1 \\
\hline 8 & -1 & -1 & -1 \\
\hline
\end{tabular}




\section{UTILITY GRAPH REPRESENTATION}

To measure the difference between the ranks of each attribute, the full factorial design has to be represented in a graphical notion known as utility graph. Since this illustration considers only three attributes, a cube is constructed as the utility graph. In this cube, the rank of each product profile is represented as a point. Figure 1 represents all the ranks in the utility graph. It represents the ranks of each product profile with their factor which is used for calculating the individual preference level of that particular attribute.

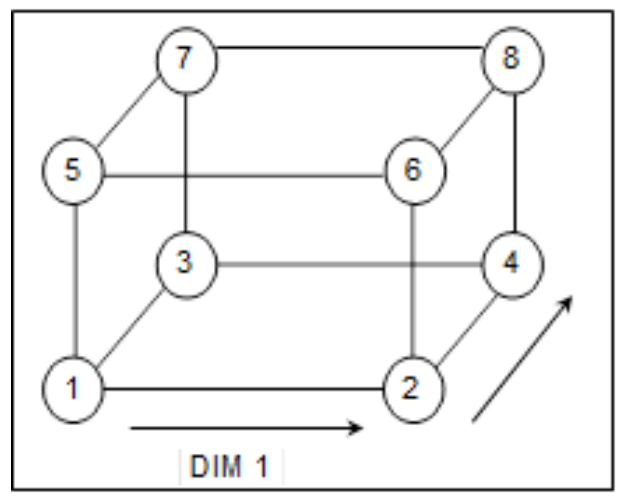

Fig. 1 Utility Graph of the factors

\section{LINEAR REGRESSION FUNCTION}

In this step, the preference of the levels of each factor are obtained such that the estimated ranks of the product profile using the utility weights highly correlate with the original ranks of the product profile.

The rank for each factorial combination is defined using the multiple linear regression function $[5,8]$ given in equation 1.1 ,

Rank $=$ Part_worth of attribute $*$ Level of attribute ${ }_{i}$

where $i=1,2, \ldots$.

Part_worth is written with an hyphen for clarity in the notation. Using equation 1.1, ranking is defined for the three factors as,

$Y=\beta_{\text {DIM1 }} *\left(X_{1}\right)+\beta_{\text {DIM2 }} *\left(X_{2}\right)+\beta_{\text {DIM3 }} *\left(X_{3}\right)+\mu$

where $\mathrm{Y}$ is rank of a product profile, $\beta_{\text {DIM1 is }}$ part_worth of Dimension 1, $\beta_{\text {DIM2 }}$ is part_worth of Dimension 2,

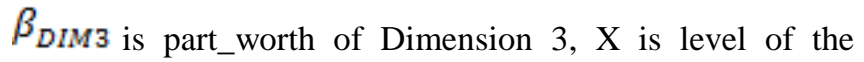
attribute and $\mu_{\text {is adjustment factor. }}$

To estimate the influence of variables on one another, a multi-variate linear regression function is derived [5]. Using the coded combinations a system of linear equations and the ranking for each combination is formed. Regression function is used in this. The linear equations for each rank are displayed in Table IV.
TABLE Iv MULTIPLE REGRESSION FUNCTION

\begin{tabular}{|c|l|}
\hline Rank & \multicolumn{1}{|c|}{ Regression function } \\
\hline 1 & $\beta_{D I M 1} *(-1)+\beta_{D I M 2} *(-1)+\beta_{D I M 3} *(-1)+\mu$ \\
\hline 2 & $\beta_{D I M 1} *(1)+\beta_{D I M 2} *(-1)+\beta_{D I M 3} *(-1)+\mu$ \\
\hline 3 & $\beta_{D I M 1} *(-1)+\beta_{D I M 2} *(-1)+\beta_{D I M 3} *(1)+\mu$ \\
\hline 4 & $\beta_{D I M 1} *(-1)+\beta_{D I M 2} *(-1)+\beta_{D I M 3} *(-1)+\mu$ \\
\hline 5 & $\beta_{D I M 1} *(-1)+\beta_{D I M 2} *(1)+\beta_{D I M 3} *(-1)+\mu$ \\
\hline 6 & $\beta_{D I M 1} *(1)+\beta_{D I M 2} *(1)+\beta_{D I M 3} *(-1)+\mu$ \\
\hline 7 & $\beta_{D I M 1} *(-1)+\beta_{D I M 2} *(1)+\beta_{D I M 3} *(1)+\mu$ \\
\hline 8 & $\beta_{D I M 1} *(1)+\beta_{D I M 2} *(1)+\beta_{D I M 3} *(1)+\mu$ \\
\hline
\end{tabular}

\section{CALCULATION OF THE PART_WORTH UTILITIES}

For each attribute, the part_worth utility is calculated. From the cube, part-worth utility is subtraction value of the summation of the rank values of all points on the right side of the vertical plane and the summation of rank values of all points on the left side of the vertical plane. Figure 2 depicts the computation of the part-worth utility for the attribute DIM1.

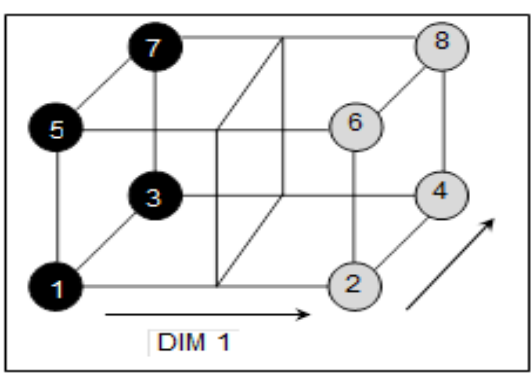

Fig. 2 Utility Graph for DIM 1

In the similar way, the part_worth utilities are calculated for other attributes as shown in Figure 3 and Figure 4.

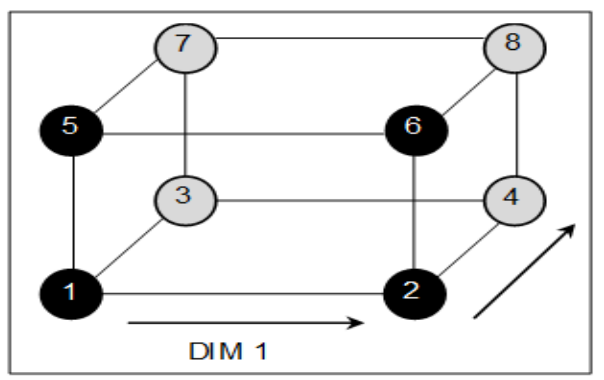

Fig. 3 Utility Graph for DIM 2

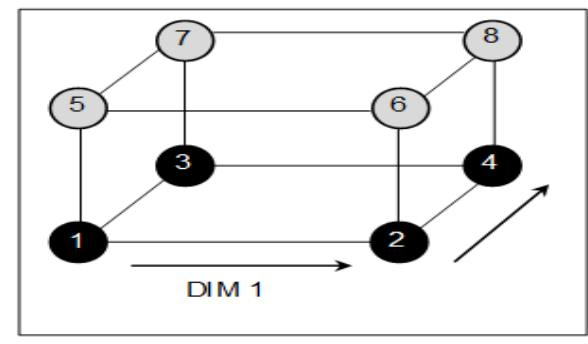

Fig. 4 Utility Graph for DIM 3 
The part_worth utilities for the other attributes are also calculated using the utility graph and the values are shown in Table V.

TABle V PART_WORTH UTILITIES
\begin{tabular}{|c|c|}
\hline Parameter & Part_worth utility \\
\hline DIM 1 & -0.5 \\
\hline DIM 2 & -1 \\
\hline DIM 3 & -2 \\
\hline
\end{tabular}

The ranks are calculated by substituting the part_worth utilities given in Table $\mathrm{V}$ in equation 1.3 as,

$Y=(-0.5) *\left(X_{1}\right)+(-1) * X_{2}+(-2) * X_{3}+4.5$

where, $\mathrm{Y}$ is the rank for a product profile and $X$ is the level of the attribute.

The utility adjustment factor $(\mu)$ is chosen such that the sum of the errors of the actual preference values and the corresponding estimated preference values is minimum [2]. When the original preference of the respondents and the estimated preferences are compared, the difference is found to be negligible. It is observed that the calculated profile ranking match the actual ranking.

\section{CALCULATION OF THE RELATIVE PREFERENCES}

The relative preference of an attribute $\left(X_{i}\right)$ can be calculated from its part-worth utility, with levels -1 and +1 , is given as,

$P_{i}=\left|v\left(X_{i}\right) * \beta_{x i}\right|$

where $P$ is an integer representing the individual preference, $v$ is the number of the levels of an attribute, $\beta_{x}$ is the part_worth utility of the attribute and $i=1,2, \ldots n$. Using equation 1.4, the preference value for each attribute is computed as an integer as given in Table VI.

\section{TABle Vi INDiVidual Preference Of The Attributes}

\begin{tabular}{|c|c|}
\hline Parameter & $\begin{array}{c}\text { Relative } \\
\text { preference }\end{array}$ \\
\hline DIM 1 & 1 \\
\hline DIM 2 & 4 \\
\hline DIM 3 & 2 \\
\hline
\end{tabular}

The total variation of all the attributes is computed using the individual preference value of the attributes and is given as,

$T=\sum_{i=1}^{n} P_{i}$

where, $T$ is the total variation of the attributes, $P$ is the variation of the individual attribute.
The relative preference of the attribute is derived using the individual preference value $(\mathrm{P})$ and the total variation $(\mathrm{T})$. From equation 1.4 and 1.5 , the relative preference is given as,

$R_{\bar{i}}=\left(\frac{P_{\bar{i}}}{T}\right) * 100$

where $\mathrm{R}$ represents the percentage of relative preference of an attribute, $\mathrm{P}$ is the preference of the attribute, $\mathrm{T}$ is the total variation of the attributes and $I=1,2,3, \ldots n$.

Using equation 1.6, relative preferences are calculated as a percentage and given in Table VII.

Table ViI Relative Preference Of The Attributes

\begin{tabular}{|c|c|}
\hline Parameter & $\begin{array}{c}\text { Relative } \\
\text { preference }\end{array}$ \\
\hline DIM 1 & $14 \%$ \\
\hline DIM 2 & $57 \%$ \\
\hline DIM 3 & $29 \%$ \\
\hline
\end{tabular}

Figure 5 presents the algorithm to find the most preferred learning style using conjoint algorithm. From the Table VII it is shown that the attribute with highest percentage value proves that it is the most influencing attribute out of the other attributes. In this case, DIM 2 is the most influential attribute comparing with DIM 1 and DIM 3.

\section{A. Algorithm Conjoint Analysis}

Input: $\quad$ Set of $m$ Parameters $P=\left\{p_{1}, p_{2}, \ldots p_{m}\right\}$.

Set of $n$ Attributes $A=\left\{a_{11}, a_{12}, \ldots a_{1 n}, \ldots .\right.$. $\left.a_{m 1}, a_{m 2}, \ldots a_{m n}\right\}$.

Set of $n$ Rankings on the Attributes

$R=\left\{r_{11}, r_{12}, \ldots r_{l n}, \ldots . r_{m 1}, r_{m 2}, \ldots r_{m n}\right\}$

Output: Set of preferences.

Set of percentage of preferences.

Set of rankings based on preferences.

1. begin

//construct the design matrix

2. Construct a design matrix with $m$ rows and $n$ columns;

3. for $i=1$ to $n$

4. $\quad$ for $j=1$ to $m$

5. DesignMatrix $[i, j]=\mathrm{A}[i, j]$;

$6 . \quad$ end for;

7. end for

//construct the utility graph and calculate the partworth utilities

8. Construct the utility graph from design matrix;

9. for $i=1$ to $m$

10. $\quad$ sumofleft $=0 ; j=1$;

11. while $(j<=m)$

12. sumofleft $=$ sumofleft $+\operatorname{rank}[i, j]$;

13. end while;

14. $\quad$ sumofright $=0 ; j=2$;

15. while $(j<=m)$

16. sumofright $=$ sumofright $+\operatorname{rank}[i, j]$; 
17. end while;

18. Part-worth $[i]=$ sumofleft + sumofright;

19. end for;

// calculate the linear regression

20. for $i=1$ to $m$

21. Ranking [i] $=$ Part - worth of attribute ${ }_{i} *$ Level of attribute $_{\mathrm{i}}$

22. end for;

//calculate the relative preferences and their percentage

23. for $i=1$ to $m$

24.

$P_{\mathrm{i}}=\left|v\left(X_{\mathrm{i}}\right) * \beta_{x \mathrm{i}}\right|$;

25.

$$
T=\sum_{i=1}^{n} P_{i}^{i}
$$

$$
R_{\mathrm{i}}=\left(\frac{P_{\mathrm{i}}}{\mathrm{T}}\right) * 100_{\text {s }}
$$

25. end for;

26. end;

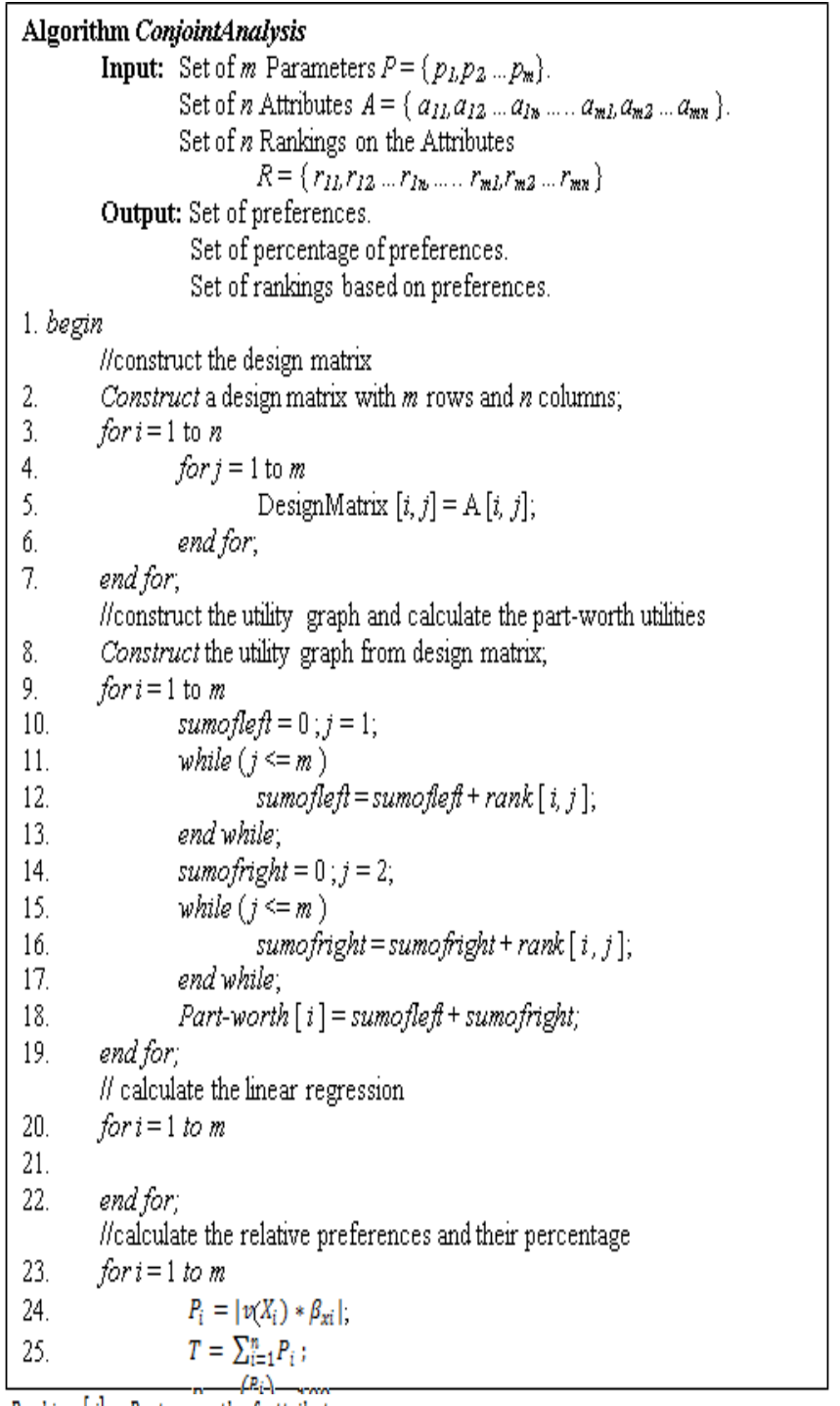

Ranking $[i]=$ Part - worth of attribute $e_{i}$ *

Level of attribute;

Fig. 5 Algorithm to find learning style using conjoint analysis

\section{EXPERIMENT}

The experiment was conducted on a group of 300 learners. Input were collected to ascertain their preferences based on the following methods

1. Conjoint Analysis

2. Maxdiff

3. Dynamic Budget Allocation

4. Simple Ranking

\section{A. Maxdiff}

The very common addition to conjoint analysis is the use of MaxDiff (also known as best-worst analysis). Respondents are told to pick the best and worst items from a small list. They are given another list and the process is repeated. This allows the items of the list to be given a utility score (and rank) in the same way as conjoint analysis. One difference is that the items are not shown in group as part of a product profile but are regarded individually relative to each other and so it looks more like a plain ranking than a full product trade off. MaxDiff is typically analyzed using a Hierarchical Bayes (HB) method with the effect that data is scaled like a conjoint study.

\section{B. Dynamic Budget Allocation}

Conjoint analysis gives lot of information since its objective is to provide a valuation for all the levels in all of the attributes. Normally we are most interested in the items that are most valuable. The way to do this as follows: first offer the levels at fixed 'point' values and then give respondents a point budget, then ask them to optimize the product within the given budget. The point values are then adjusted and the respondent repeats the task mainly discarding the items not chosen, while focusing on the items that are of most value.

\section{Simple Ranking}

Ranking questions also force individuals to trade-off between alternatives. Ranking is one method of collecting data from a simple full-profile conjoint analysis. Ranking is traditional and tiresome with more than about 8 or 10 items. In this tasks can be split or rotated just to simplify the task. The use of online surveys allows ranking to be done more easily - eg a click-to-rank, drag-and-drop or by asking respondents to make selections in roughly rank order and monitoring mouse clicks.

The learners were asked to use the content of the e-learning system with all types of media (text, audio, video, presentations, etc.). The time the learners have taken on every learning content and the frequency (count) of the learning resources used were collected using the model presented below. This model analyses the continuously captured data pertaining to the usage of e-learning components by the learners. Any e-learning system is very dynamic in terms users, components and the usage behavior of the users. A comprehensive system is required to capture 
every event and the change of state initiated by the introduction and removing of e-learning components and introduction and removing of users. The model is explained below.

Let $U_{l}$ be the utilization matrix of the learner $l$, ranging from $l$ to $n$.

Let $x$ be the number of rows of numbered from 1 to $x$.

Let $y$ be the number of rows of numbered from 1 to $y$.

$x=y$

Let $c_{j}$ be the learning content $j$.

Let $t_{j}$ be the time at which the content $c_{j}$ was available in the system.

Let $U_{l i j}$ be the percentage of time spent by the learner $l$ on the content $c_{j}$ during the interval $t_{i}$ and $t_{i+1}, t_{k}=0$ if $k<1$.

Conversely, $U_{l i j}$ is the percentage of time spent by the learner $l$ on the content $c_{j}$ during period between the introduction of the contents $c_{i}$ and $c_{i+1}$.

$U_{l i j}=0$ if $j>i$.

Let $U^{\prime}$ be the utilization matrix of all the learners.

Let $U_{i j}^{\prime}$ be the statistical summary of all $U_{l i j}, l$ ranging from 1 to $n$.

For example, $U_{i j=}^{\prime} \frac{\sum_{p=1}^{\mathrm{n}} U_{p i j}}{n}$ gives the average time of all the learners spent on content $c_{j}$ in the interval $t_{i}$ and $t_{i+1}$.

Let $P_{i j}$ be the probability distribution of $U_{l i j}, l$ ranging from $l$ to $n$.

The data that is captured is basically multidimensional and any multidimensional analysis can be applied on it to derive the desired results. The highlight of the above model is that it transforms into an underlying data structure that is an ndimensional lower triangular matrix that reduces the computational complexity. In this research, multidimensional regression and correlation models have been employed extensively for the purpose of analysis at each time interval. Moreover, moving windows and weight based analysis technique were used for augmenting the regression and correlation analysis to consider more relevant dimensions and components and arrive at realistic interpretations of the user behavior. The spatial and temporal proximities of the components that fall within the window were given more weightage in the analysis.

Based on the observed results, graphs were drawn. The following graphs depict the accuracy of the methods based on time spent and frequency of learning content usage. The results are shown in Figure 6 and Figure 7.

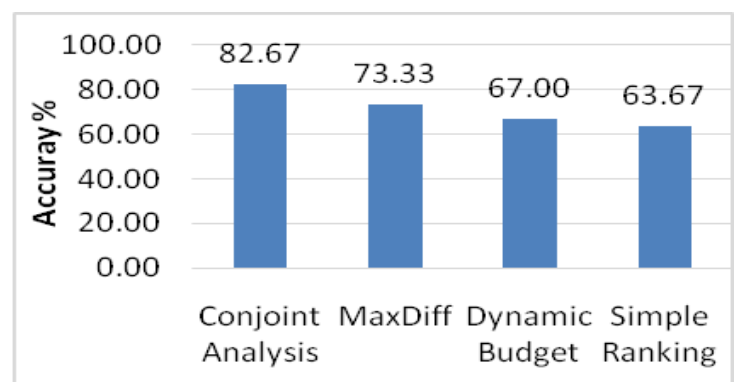

Fig. 6 Preference Prediction Accuracy of various Methods based on Time

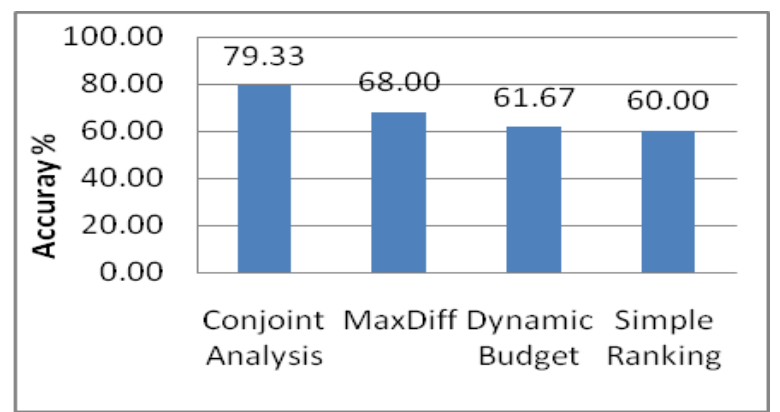

Fig. 7 Preference Prediction Accuracy of various Methods based on Frequency

\section{CONCLUSION}

The conjoint analysis is used to find the most influencing parameter from a group of attributes. Finally the result is represented as percentage. This percentage represents the prominent learning style of the individual learner. The percentage value of each dimension is clearly given. The Dimension with the highest percentage indicates the dimension that suits the individual learner and subsequently shows the most preferred learning style of the learner.

The following conclusions can be made as a result of the experiment carried out on the learners.

1. The results computed using conjoint analysis and those obtained from the experiment are almost matching. There is an accuracy of $82.67 \%$ based on time factor and $79.33 \%$ on frequency factor. The theoretical calculations are confirmed by experimental results.

2. The computed results and the experimental observations prove that conjoint analysis method has got far greater accuracy in terms of time and frequency $(82.67 \%$ \& $79.33 \%)$, than the other methods, namely, Maxdiff $(73.33 \%$ \& 68.00\%), Dynamic Budget Allocation $(67.00 \%$ \& $61.67 \%)$ and Simple Ranking (63.67\% \& 60.00\%).

\section{REFERENCES}

[1] R. Panneerselvam, Research Methodology, Second Edition, PrenticeHall of India, New Delhi, 2014.

[2] A. Hamdy Taha, Operations research: an introduction, Prentice Hall of India, New Delhi, 1999.

[3] S. C. Gupta and V. K. Kapoor, Fundamentals of Mathematical Statistics, Sultan Chand \& Sons, New Delhi, India, 2014.

[4] K. Chrzan and B. Orme, "An Overview and Comparison of Design Strategies for Choice Based Conjoint Analysis", Sawtooth Software Research Paper Series, 2000.

[5] R. VithalaRao, Applied Conjoint Analysis, Springer, 2014.

[6] D.Agbenyegah, "Tell Me What You Want: Conjoint Analysis Made Simple Using SAS", SAS Global Forum, Paper 3042, 2015.

[7] B. Vermeulen, P. Goos and M. Vande broek, "Models and optimal designs for conjoint choice experiments including a no choice option", International Journal of Research in Marketing, Elsevier, Vol. 25, No. 2, pp. 94-103, 2008.

[8] D. Raghavarao, B. James Wiley and P. Chitturi, Choice-Based Conjoint Analysis, Models and Designs, Taylor and Francis Group, 2010. 